\title{
Experimental study of a 3D dry granular medium submitted to horizontal shaking
}

\author{
A. RAIHANE(1), O. BONNEFOY(1*), J --L. GELET(2) , J .-M. CHAIX(3), G. THOMAS(1) \\ (1) Département PMMC (Poudres et Matériaux Multi Composants), Centre SPIN - LPMG \\ UMR CNRS 5148. Ecole Nationale Supérieure des Mines de Saint-Etienne. 158, Cours \\ Fauriel. 42023 Saint-Etienne Cedex 2. \\ (2) Ferraz-Shawmut. 6, rue de Vaucanson. 69720 Saint-Bonnet de Mure. \\ (3) SIMAP-LTPCM, INPGrenoble-CNRS-UJF. BP 75 - Domaine Universitaire. 38402 Saint- \\ Martin d'Hères \\ (*) obonnefoy@emse.fr \\ keywords:
}

Horizontal vibrations; Sand granular medium; Fluidization; Convection; Steady state

\section{Abstract}

This experimental work consists in a study of the influence of horizontal sinusoidal vibrations on the behaviour of a $3 \mathrm{D}$ sand granular medium. A transition from jammed or glassy states to a fluidized state has been observed for critical acceleration increasing with the frequency. Convective patterns have been encountered and characterised: two cylindrical counter-rotating rolls have been observed in the top of the packing. In addition, for high acceleration values, more complex patterns appear.

The free surface at the top of the packing presents different shapes called "dome", "dromedary" or "camel" depending on acceleration. One or three humps can be found. Analysis of grain movements and velocities along the walls of the container has been carried out and show that a stationary state can be reached after a transient regime. Finally the mechanisms of material transport through convective motion have been discussed.

\section{Introduction}

Vibrations applied to granular media are widely used in industry to improve powder flowability, to prepare homogeneous mixtures or to give a better separation of materials with respect to their size, density. For example, periodic shocks or vibrations applied to granular media can increase density and ensure satisfactory filling in packaging, and as used in electrical fuses. The maximum possible compacity is targeted to improve the fuse cut efficiency.

So, many studies have been developed to obtain a better understanding of vibrated granular media. Most have been carried out with vertical vibrations [1], [2] and [3] and only a few with horizontal vibrations. These latter studies have been mainly performed in 2D or quasi-2D geometry, i.e. the dimension of the wall parallel to the vibration direction being much smaller than the dimension at right angles to it. Experimental and numerical studies have been focused on two phenomena: the transition from a solid to liquid behaviour [4], [5], [6] and [7] and the convective movements [8], [9], [10] and [11]. The present study concerns horizontally shaking granular media in a full $3 \mathrm{D}$ geometry.

First, the experimental setup will be described. Then, different phenomena observed experimentally will be presented, and in particular, the transition to fluidization and the convective patterns. The shape of the free surface will also be described. Finally, a quantitative determination of the time evolution of the grain velocity field will be proposed as well as an estimation of the minimal delay necessary for the vibrated granular medium to reach a dynamic steady state from the static conditions. 
Experimental setup and materials

The experimental assembly consists in a mobile horizontal table, on which a container is fixed and partly filled in with the granular medium (Fig. 1).

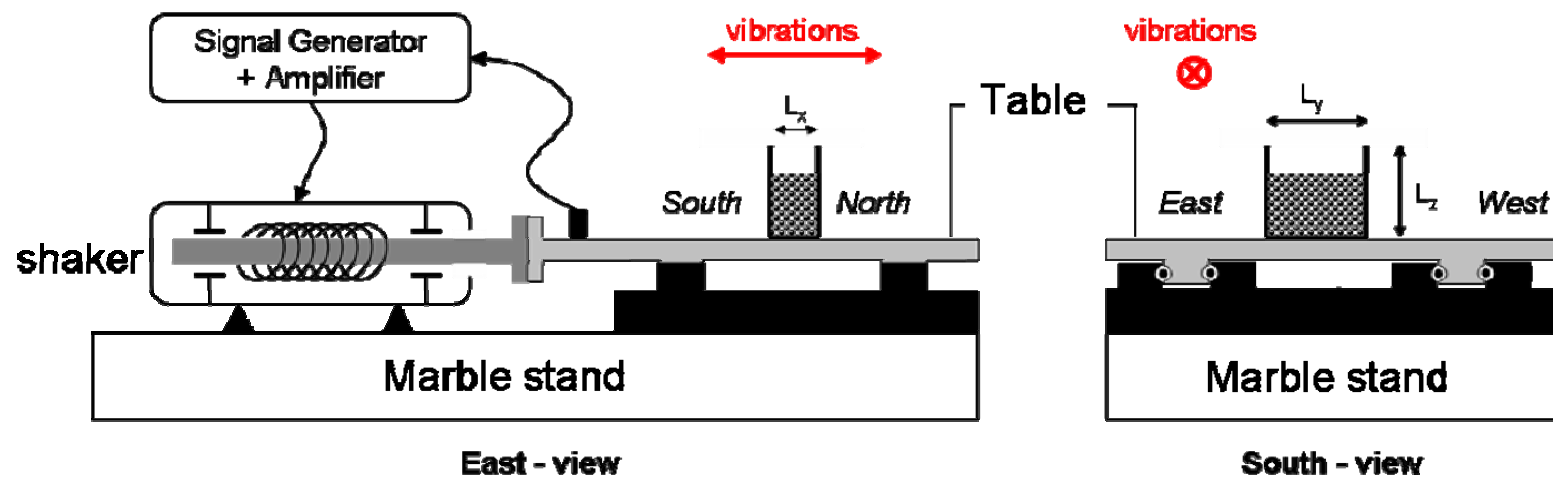

Fig. 1: Experimental setup : the electromagnetic shaker drives the table which is mounted on four bearings and guided by four rails. The sand container is a transparent Plexiglas box fixed on the mobile table. The direction of shaking is referred as $\mathrm{x}$ and also as the south-north axis.

A table made of an aluminium alloy is linked to a marble stand by four horizontal linear motion guides (THK, EPF7M16 + 55LM). The motion is transmitted by an electromagnetic shaker (TIRA, S513) driven by a signal generator (LING DYNAMIC SYSTEMS, DSC4) coupled with a power amplifier (TIRA, BAA120). The motion is driven with an accelerometer (BRUEL and KJAER, $4371 \mathrm{~V}$ ). When the feedback option is activated, the power is constantly adjusted to compensate any phase difference or dampening caused by friction or similar disturbing phenomena.

In addition, a visualization accelerometer (TIRA, DeltaTron 4517) with an accuracy of $1 \%$ between $10 \mathrm{~Hz}$ and $10 \mathrm{kHz}$, and an ultra-fast CCD camera (JAI, CMOS CV-A33) with a maximum frequency of 5400 images/s and a maximum window of $494 \times 660$ pixels allow us to quantify the vibrations applied to the container and follow the movements of grains along the walls of the box.

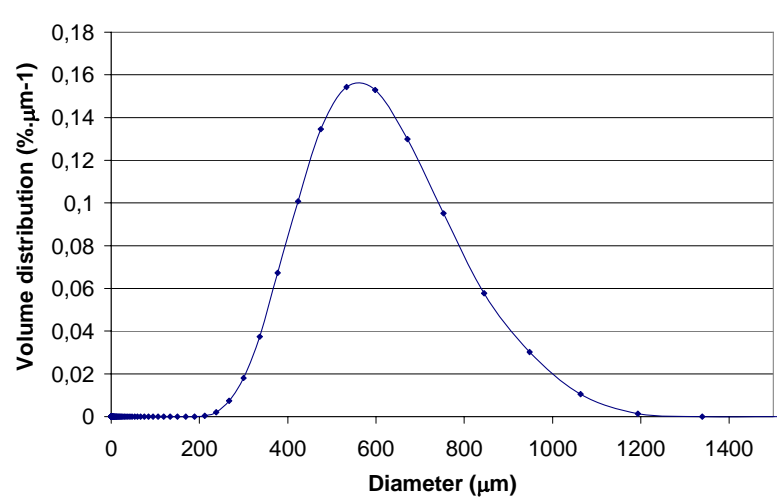

Fig. 2: Grain size distribution: the mean diameter $\mathrm{d}_{4,3}=575 \mu m$ and the span $=\left(\mathrm{d}_{90 \%}-\mathrm{d}_{10 \%}\right) / \mathrm{d}_{50 \%} \sim 0.75$ (where $\mathrm{n} \%$ in mass of the grains have a diameter lower than $\mathrm{d}_{\mathrm{n} \%}$ ).

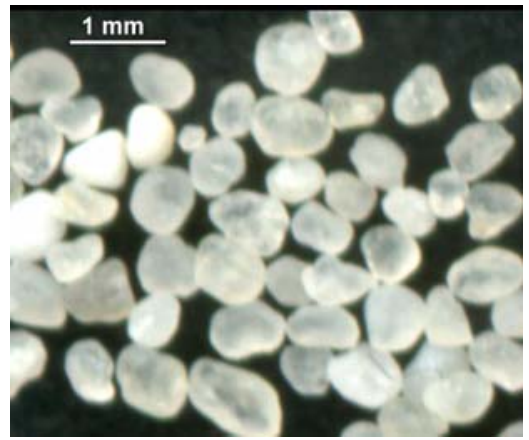

Fig. 3: Sand grain morphology: microscope photograph showing smooth rounded grains.

The container is a parallelepiped composed of an assembly of screwed or glued plates, with a thickness which varies between 6 and $10 \mathrm{~mm}$. The wall-grain friction is an important factor under horizontal vibrations. To get a better simulation of industrial conditions, as the industrial fuse-holder has smooth walls, smooth plates made of Plexiglas have been chosen, which also make easier the observation of the grain motion. The container itself is screwed or 
glued (cyano-acrylate) to the mobile table. The dimensions $L_{x}, L_{y}, L_{z}$ of the volume available for the granular medium are respectively 40,80 and $80 \mathrm{~mm}$. The initial height of the granular packing is $60 \mathrm{~mm}$.

The grain material is sand with more than $99 \%$ of alpha-quartz and a density of $2660 \mathrm{~kg} / \mathrm{m}^{3}$. The size distribution given on Fig. 2 shows a moderate polydisperse material ( span $=0.75$ ) and a mean diameter of $\mathrm{d}_{4,3}=575 \mu \mathrm{m}$. The grains are approximately spherical (Cf. Fig. 3).No internal close porosity has been detected: the pycnometer density determination shows that the grain density corresponds to the perfect crystal. The packing porosity corresponds to inter-particle pores.

\section{Experimental protocol}

To fill in the container, the sand is poured through a funnel (diameter $\sim 1 \mathrm{~cm}$ ) that is moved to scan the whole container surface. This method leads to a compacity of $62 \% \pm 1 \%$. When the container is filled with the desired amount of grains, the shaker drives the $<$ table + container + grains $>$ system with sinusoidal vibrations of amplitude $A$ and frequency $f$ along the $O x$ direction. The relative acceleration $\Gamma$ is defined by:

$$
\Gamma=A .(2 \pi f)^{2} / g \text { where } g \text { is the vibrational acceleration }
$$

In the experiments described in this article, the frequency is comprised between 20 and $300 \mathrm{~Hz}$, the relative acceleration in the range 0.1 and 8 and the amplitude between 0.3 and $3000 \mu \mathrm{m}$, that is to be compared with the grain mean diameter $\mathrm{d}_{4,3}=575 \mu \mathrm{m}$.

\section{Characterisation methods}

Through the experiments, one can estimate the packing fraction also called compacity or relative density (i.e. solid volume fraction) and the grain movements (displacement and velocity) at the external faces of the container.

\section{Compacity}

The global compacity can be defined as the ratio of the grain volume to the apparent volume (grain volume + pore volume). By measuring the shape of the free surface, the global compacity may be calculated in two cases: in the static mode (i.e. before or after the vibrations) and in the dynamic mode (i.e. during the vibrations) when the excitation is such that the free surface possesses a well defined shape.

\section{Grain movements}

Along the walls, the observation of the grains movements during vibration is facilitated by dying with Indian ink the naturally light yellow grains. To establish the grain velocity field of the granular medium along a wall, an ultra-fast acquisition camera has been used to obtain video records of grain movements and analyzed after by ImageJ software by tracking grains individually on successive photographs.

\section{Experimental results}

\section{Steady-state regime}

In the literature, it is commonly admitted that a granular medium may present the three following states:

* Jammed: the contact network is not disturbed by the vibrations. The granular medium behaves like a rigid solid, even over long timescales.

* Glassy: grains are animated by a slight chaotic movement, analogous to Brownian diffusion, and with an amplitude i much smaller than the grain size. Grains are trapped in cages formed by the other grains and can rarely escape. Under these conditions and with gravity acting, a densification may be observed, as well as a slight simmering of the grains at the free surface. 
* Fluid: the mean free path is bigger than a grain size and the granular medium behaves like a fluid. If a driving force like gravity and/or vibrations is present, convective motion may appear, with a complexity level depending on the geometry, grain characteristics and driving force parameters.

In our experiments, two of these three states have been observed, often simultaneously. In fact, under certain vibratory conditions, the granular medium state is not uniform in the container and many zones can appear with a defined state each. Fig. 4 sums up some experimental results obtained with our apparatus along the vertical axis dividing the North face into two halves.

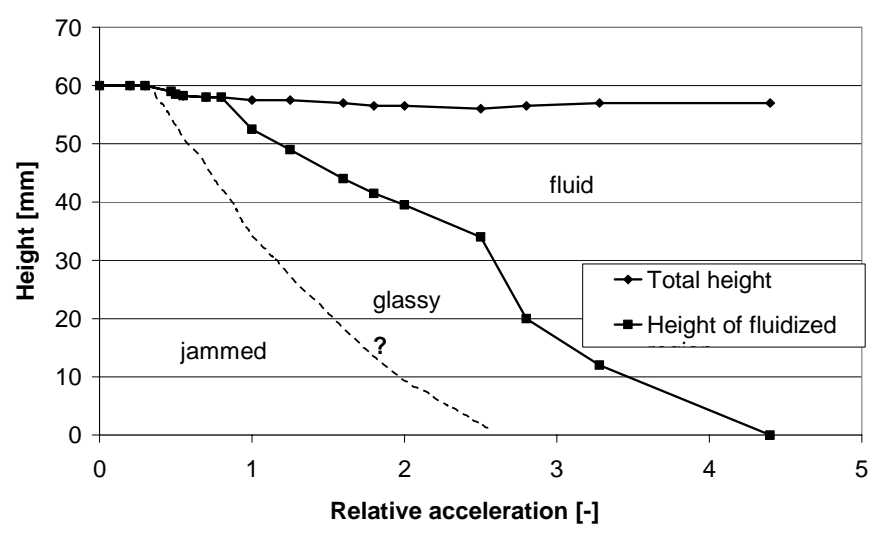

Fig. 4: Granular state diagram: transition curves between the three states of the granular sand vibrated at $\mathrm{f}=50 \mathrm{~Hz}$. The solid curve: observed fluid-glassy transition. The dotted curve: jammedglassy hypothetic separation. Observations made in the middle of the north face.

For $\Gamma<0.4$, the dynamic global compacity does not change with time. From this and because no motion is observed, we deduce that the granular medium is uniformly jammed.

For $0.4<\Gamma<0.8$, the total height decreases by $10 \%$. Compacity measurements in the relaxed state (i.e. at rest after vibrations) show that this densification only occurs in the upper part of the packing. Hence, one can imagine that the granular medium remains jammed in the lower part and becomes glassy in the upper part. However, the separation line between these two zones cannot be determined with our experimental setup (dotted line on Fig. 4). On top of the granular medium, the grains are simmering randomly over a depth of two grain diameters $(\sim 1 \mathrm{~mm})$.

For $\Gamma>0.8$, above the jammed and glassy zones, a fluidized layer can be seen, with a thickness which increases with acceleration. There, the grains are dragged in convection rolls, with a rotation period of around $60 \mathrm{~s}$ for $\Gamma$ equal to 2. As shown on Fig. 5, the rolls remain invariant per translation along the transverse Oy axis when $\Gamma<4$. For bigger relative accelerations, the presence of the East and West faces alter the rolls shape. Moreover, the convection motion concerns the whole packing. In Fig. 5 it can be seen that counter-rotating rolls have a size of half the container width, and its thickness corresponds to the thickness of the fluid zone seen at the north wall. Roughly a mean radius can be proposed to define the largest top roll as a cylinder of radius $10 \mathrm{~mm}$ and length $80 \mathrm{~mm}$ with the chosen container.

The shape of the free surface is also affected by the acceleration. As shown on Fig. 6, the surface is plane for $\Gamma<0.7$ and it forms a slight dome for $0.7<\Gamma<2$. For $2<\Gamma<4.4$, a central summit line with two adjacent intermediate lines can be seen ("camel"). These three lines merge into a single one ("dromedary") for $\Gamma>4.4$. 


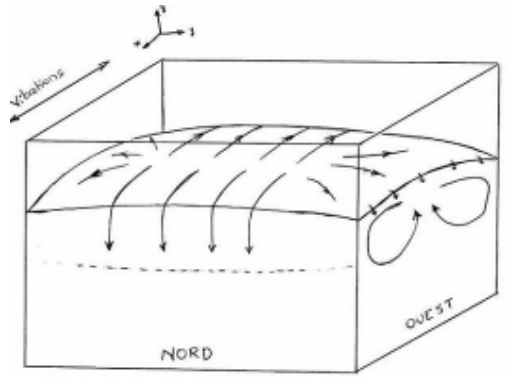

Fig. 5a: Convection rolls for $0.8<\Gamma<4$ and $\mathrm{f}=50 \mathrm{~Hz}$ : two-rolls in the upper part; the dotted line on the north face indicates that grains disappear in the core of the granular bed. It is also the separation zone between the fluidized region and the glassy/jammed region: 2-rolls in the upper part; the dotted line on the north face indicates that grains disappear in the core of the granular bed.

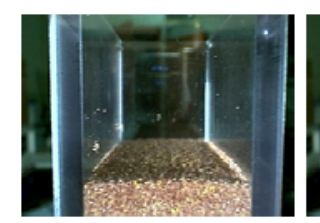

$1=1$

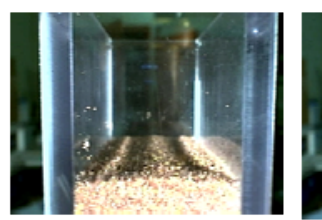

$\underline{1}=2,5$

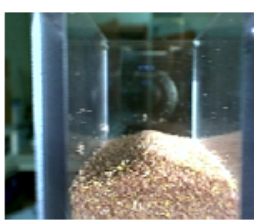

$I^{\prime}=4,4$
Fig. 6a: Shape of the free surface of the sand packing at $\mathrm{f}=5 \mathrm{O} \mathrm{Hz}$ viewed from the west face.

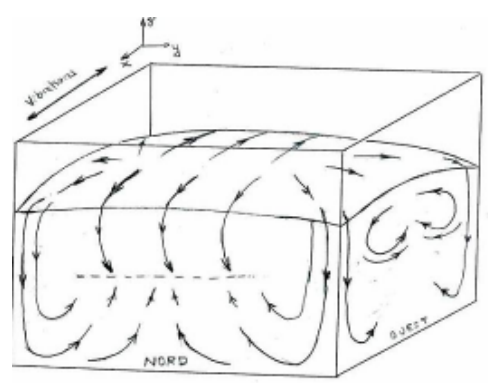

Fig. 5 b: Complex convection patterns for $\Gamma>4$ and $\mathrm{f}=50 \mathrm{~Hz}$. In addition to the two counter-rotating rolls in the upper part, the strong fall at the corners induces visible upwards convection on all faces.

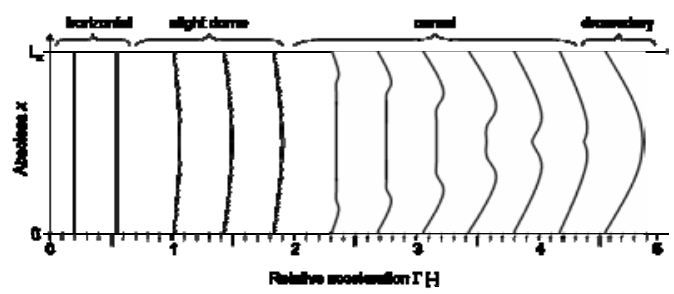

Fig. 6 b: Evolution of the free surface with $\Gamma$ at $\mathrm{f}=50 \mathrm{~Hz}$ observed from the west face.

Moreover, the grains at the free surface move from the median (yz) plane towards the walls with a homogeneous velocity. The latter is low in the central zone and increases suddenly when leaving the humps. The difference between the two fields of velocity increases with acceleration.

Getting vibrated: characterizing the transient regime

Submitted to vibrations, the system initially at rest reaches a quasi-stationary state after a certain time-lag that we determined in two shaker configurations: with and without feedback loop. The experiments are carried out with sinusoidal vibrations $(f=50 \mathrm{~Hz}$ and $\Gamma=2.2)$.

Fig. 7 shows that the presence of the grains does not noticeably change the container's movement. This means that the shaker delivers a power great enough to ensure the prescribed movement to the container whatever the content. The stationary regime is reached after $30 \mathrm{~s}$ when the feedback loop is activated and otherwise after only few seconds. The control quality in the stationary regime is slightly better with the feedback loop.

To measure the dependence of the grain velocity upon time, an ultra-fast CCD camera synchronized with the electromagnetic shaker has been used. More precisely, we chose to measure the vertical component of the grain velocity in two different windows on the North face for $\mathrm{f}=5 \mathrm{O} \mathrm{Hz}$ and $\Gamma=2.2$ (see Fig. 8). The velocities are averaged on at least 10 pairs of frames, and for each pair, on about 10 grains in the window. 


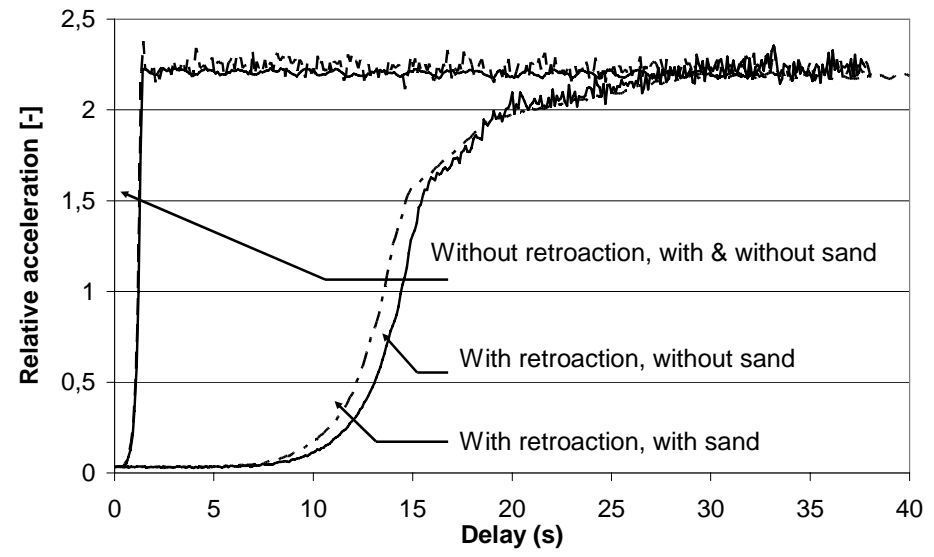

Fig. 7: Transient and steady-state regimes. Influence of the shaker control type and the mass in the container for $\mathrm{f}=50 \mathrm{~Hz}$ and $\Gamma=2.2$.

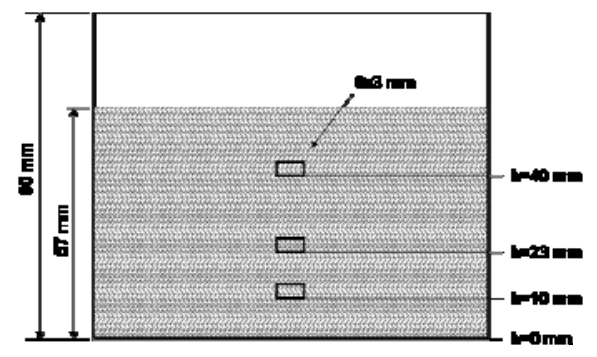

Fig. 8a: Analyzing grain movements in two zones at the north face.

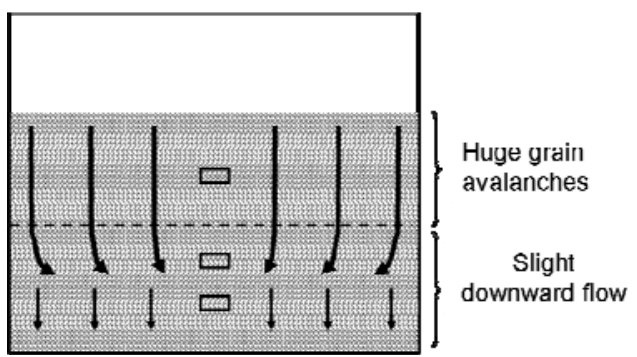

Fig. 8 b: Flow patterns of particles on the north face for $\mathrm{f}=50 \mathrm{~Hz}$ and $\Gamma=2.2$.

Figure 9 shows the grain velocity. In spite of the dispersion associated with the chaotic nature of the grain movements, trends are clearly visible. The grain velocity progressively increases with time and stabilizes at a constant value depending on the altitude. The duration of the transient regime is of the order of $30 \mathrm{~s}$, which has already been observed for the amplitude of the container movement. Terminal velocities range between 0.1 and $3 \mathrm{~mm} / \mathrm{s}$, which corresponds to the mean grain displacements of the order of 2 to $60 \mu \mathrm{m} /$ cycle of $20 \mathrm{~ms}$ $(f=50 \mathrm{~Hz})$, i.e. 0.003 to 0.1 grain diameter per cycle.

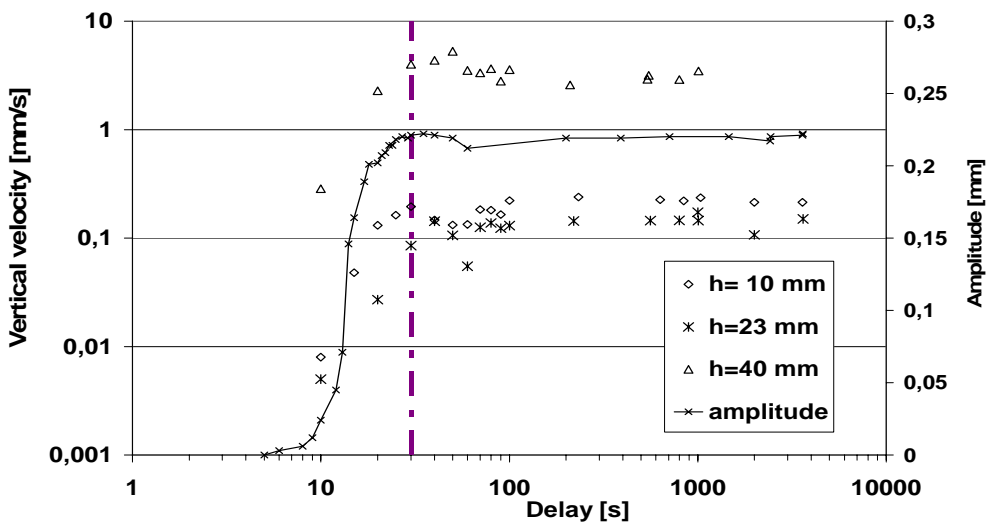

Fig. 9: Transient regime with the feedback loop forf $=50 \mathrm{~Hz}$ and $\Gamma=2.2$. The vertical velocities of grains on the north face for two different altitudes. The evolution of the amplitude of the electromagnetic shaker vs. time is also plotted. 
For a relative acceleration of 2.2 and a frequency of $50 \mathrm{~Hz}$, we have seen that a stationary regime (both for the container and for the grains) is established after 30 seconds when the feedback loop is activated. For frequencies and accelerations in the domain studied, the stationary state is considered reached after a time-lag of 100 seconds.

\section{Discussion}

\section{Grains movements in stationary regime}

On the basis of the observations reported on Figs. 4 and 6 , three transitions may be defined:

* Jamming-glassy transition: when the relative acceleration goes beyond $\Gamma_{\mathrm{c} 1} \sim 0.4$, a part of the jammed packing becomes glassy (i.e. surface simmering and core densification)

* Convection transition: for relative acceleration above $\Gamma_{\mathrm{c} 2} \sim 0.8$, convection motions are observed

* Camel-dromedary transition: above $\Gamma_{\mathrm{c} 3} \sim 4.4$, the three summit lines merge to one.

Let us point out that all transitions are smooth i.e. the state of the granular medium varies continuously with $\Gamma$ and f. However, when different zones coexist with a different state each, the intermediate regions' size can be sometimes only a few grain diameters broad.

With the experimental tools at our disposal, the first transition has not been clearly detected. We think that the third transition is a consequence of the convective patterns evolution when the acceleration increases. Now, we will focus only on the mechanisms underlying the convection transition.

\section{Convection transition}

The transition from glassy to fluidized state is difficult to observe experimentally. In effect, for a given frequency, an acceleration increase leads to a progressive increase of the mean free path of the grains and a simple visual observation does not allow distinguishing between grains entrapped in dynamic cages and freely but slowly moving grains. However, in the fluidized state, it is easier to distinguish the absence or the presence of convective motion.

For $\Gamma<4$, the convective rolls are symmetric and one can study the mechanisms in a $2 \mathrm{D}$ geometry. By image analysis, the trajectories followed by the grains at the East wall have been established (see Fig. 10a).

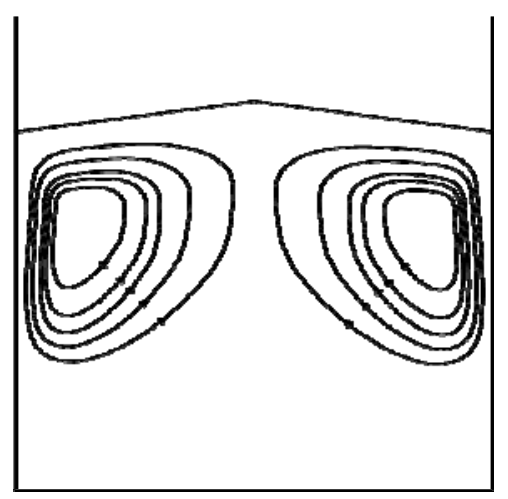

Fig. 10a: Schematic $2 D$ - representation of convection rolls seen on the east face. Grains fall down near the north and the south faces, and rise up on the middle of the cell.

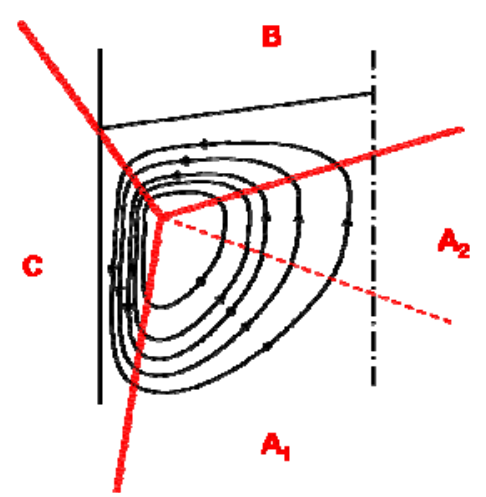

Fig. 10 b: Zoom on a convection roll. Four different zones can be differentiated according to the motion direction or origin.

Figure 1ob shows that different motions coexist: 
* Zone A: grains move to the walls, avalanching on the slope in a layer of only a few grain diameters. Compared to static conditions, the existence of vibrations makes the heap angle much smaller for $\Gamma$ and f near the transition conditions.

* Zone B: grains fall down under gravity in voids created periodically between the north or south wall and the packing. The vibration energy must be sufficiently high to create these voids along the walls.

* Zone $\mathrm{C}_{1}$ : grains coming from $\mathrm{B}$ fill in the void and, consequently, push the grains horizontally towards the middle of the packing.

* Zones $\mathrm{C}_{1}$ and $\mathrm{C}_{2}$ : grains are moving upwards.

The presence of an ascending vertical movement in a configuration, where the vibrations are horizontal and the gravity attracts grains downwards deserves an explanation. To our mind, two elements are required. The first is the ability of some granular media to reorientate the forces. In our case, for geometrical reasons, when two spherical grains are compressed by two others (along the horizontal axis), they are submitted to an orthogonal force (along the vertical axis ( $C f$. Fig. 11a) under the conditions such that an open space is free for allowing the displacement of the grain \#1 or \#2 along the $z$ axis (Fig. 11b).

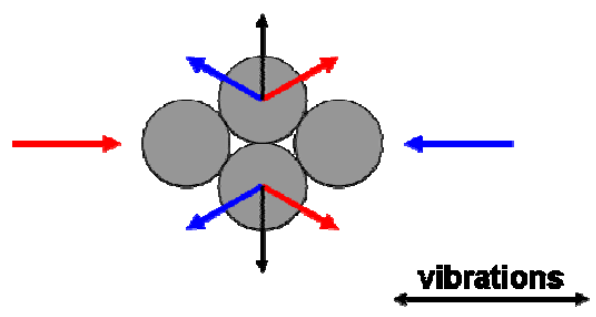

Fig. 11a: Force reorientation in the vibrated packing.

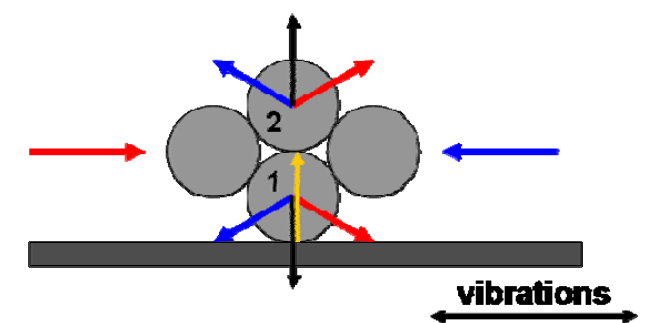

Fig. 11 b: Wall effect: only the grain \#2 will move upwards.

However, it is not sufficient to explain the ascending movement since there is no reason to define a privileged direction along this orthogonal axis. Let us now consider a virtual experiment, without gravity or on a horizontal plane, where grains are in contact with a rigid wall. Fig. 11b shows that the reaction force exerted by the wall on grain \#1 annihilates the net force normal to the bottom wall. Hence, this grain does not move. However the net force along the same axis acting on grain \#2 is different from zero and this grain can move away from the wall.

So, the ascending movement of the grains is not directly related to gravity but rather to the presence of a wall or, more generally, of a rigid base (for instance jammed grains). When vibrations parallel to this wall are applied grains tend to move away from it.

This convection transition has been experimentally studied by Behringer [4] and [5]. According to these authors, this transition depends mainly on the acceleration and is almost independent of the frequency. However, they operated with frequencies between 7 and $15 \mathrm{~Hz}$ and their conclusion must be re-examined and discussed in our case (frequency 20 to $300 \mathrm{~Hz}$ ). As shown on Fig. 12, the acceleration at the transition point clearly increases with the frequency. These results are consistent with the work of Aumaitre [7]. Moreover, we noticed that the nature of the transition depends on the frequency. For instance, for high frequencies $(\sim 300 \mathrm{~Hz})$, we no longer observe the simmering at the free surface. The whole packing seems jammed for relative accelerations below the critical value $\Gamma_{\mathrm{c}} \sim 1.5$. Above this value, some grains fall down along the walls and the convective motion starts. Let us notice that the vibrations amplitude is about $50 \mu \mathrm{m}$ for $f=50 \mathrm{~Hz} / \Gamma=0.5$ and it is about $4 \mu \mathrm{m}$ for $f=300 \mathrm{~Hz} / \Gamma=1.5$. 


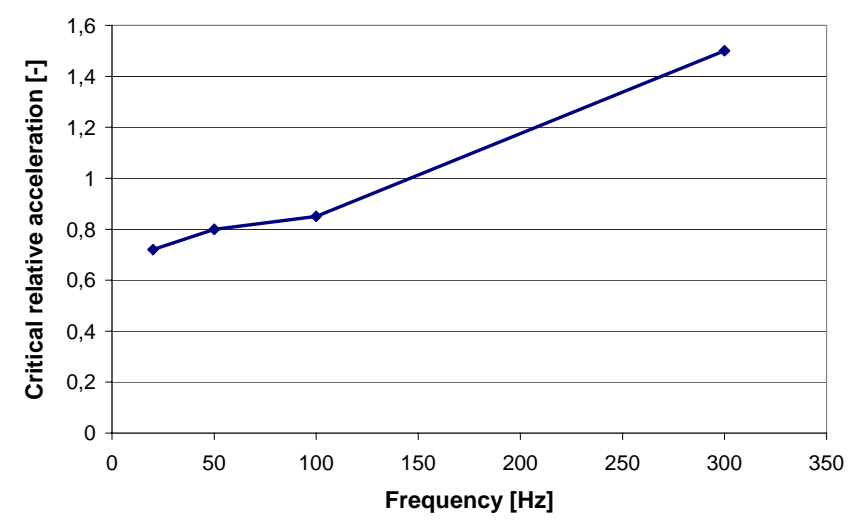

Fig. 12: Dependence of the convection transition on the frequency. Critical relative acceleration increases with the frequency of the vibrations.

\section{Conclusions}

In a $3 \mathrm{D}$ geometry, a horizontally shaked granular medium shows different behaviours when sinusoidal vibrations are imposed. With increasing acceleration, the packing segregates in potentially three zones: a jammed lower part, a glassy intermediate part and a fluidized upper part. The acceleration required for the presence of a fluidized part increases with the frequency. In this region, counter-rotating rolls can be encountered and their characteristics (size, rotation period) for different acceleration levels can be evaluated.

The shape of the free surface undergoes various transitions with increasing acceleration. The summit of the free surface is located at the arrival of the particles flowing from the bottom to the top in the central part (core) of the packing thanks to convective rolls. For instance, at $50 \mathrm{~Hz}$, we observe humps that merge into a single one above an acceleration of 4.4 g. The mechanism of this transition called 'camel-dromedary transition' needs to be further studied.

The time evolution of the grains velocity along the North face has been measured and, despite some dispersion, shows that a steady state regime is reached after a time depending on the vibration parameters (about $30 \mathrm{~s}$ for a feedback loop vibration control, frequency $50 \mathrm{~Hz}$, and acceleration $2.2 \mathrm{~g}$ ).

\section{REFERENCES}

[1] S. Fauve, S. Douady and C. Laroche. Collective Behaviors of Granular Masses under Vertical Vibrations. Journal De Physique, 1989. 50(C-3) : p. 187-191.

[2] J.B. Knight et al. Experimental study of granular convection. Physical Review E, 1996. 54(5) : p. 5726-5738.

[3] E. Rouèche, G. Thomas, J.L. Gelet, J.M. Missiaen. Dependence of granular arrangements under vibrations on the dimensions of cylindrical containers. Powder \& Grains, Vol. 2, Ed. R. Gardia-Rojo, H.J. Herrmann and S. Mc Namara, Balkema (2005), p. 1177-1180.

[4] S.G.K. Tennakoon, L. Kondic and R.P. Behringer. Onset of flow in a horizontally vibrated granular bed : Convection by horizontal shearing. Europhysics Letters, 1999. 45(4) : p. 470-475.

[5] G. Metcalfe et al. Granular friction, Coulomb failure, and the fluid-solid transition for horizontally shaken granular materials. Physical Review E, 2002. 65(3).

[6] G.H. Ristow, G. Strassburger and I. Rehberg. Phase diagram and scaling of granular materials under horizontal vibrations. Physical Review Letters, 1997. 79(5) : p. 833836. 
Powders and Technology, 2009, 190(1-2), 252-257, doi:10.1016/j.powtec.2008.04.068

[7] S. Aumaitre, C. Puls, J.N. McElwaine, J.P. Gollub. Comparing flow thresholds and dynamics for oscillating and inclined granular layers. Physical Review E, 2007, Art. no 061307

[8] K. Liffman, G. Metcalfe and P. Cleary. Granular convection and transport due to horizontal shaking. Physical Review Letters, 1997. 79(23) : p. 4574-4576.

[9] C. Saluena and T. Poschel. Convection in horizontally shaken granular material. European Physical Journal E, 2000. 1(1) : p. 55-59.

[10] M. Medved et al. Convection in horizontally vibrated granular material. Chaos, 1999. 9(3) : p. 691-696.

[11] S.S. Hsiau, M.Y. Ou and C.H. Tai. The flow behavior of granular material due to horizontal shaking. Advanced Powder Technology, 2002. 13(2) : p. 167-180. 